\title{
Screening for germline DND1 mutations in testicular cancer patients
}

\author{
Rolf H. Sijmons · Yvonne J. Vos · Johanna C. Herkert • Krista K. Bos • \\ Martijn F. Lutke Holzik · Josette E. H. M. Hoekstra-Weebers • \\ Robert M. W. Hofstra $\cdot$ Harald J. Hoekstra
}

Published online: 22 April 2010

(c) The Author(s) 2010. This article is published with open access at Springerlink.com

\begin{abstract}
Although several observations suggest that a strong genetic predisposition to developing testicular germ cell tumors (TGCT) exists, no associated, highly penetrant germline mutations have been identified so far. In the 129/ $\mathrm{Sv}$ mouse strain, a germline mutation in the DND1 gene has been shown to strongly increase the TGCT risk. We screened 272 men with TGCT (89\% sporadic cases, $11 \%$ familial) for germline mutations in the human homologue of DND1. A single nucleotide substitution c.657C > G (p.Asp219Glu) was observed in a non-familial case of testicular embryonal carcinoma. The variant was also present in the patient's asymptomatic father and two brothers, but not observed in 210 control chromosomes. The wild type DND1 allele was not lost in the patient's tumor. In silico analysis of the variant predicts it to be nonpathogenic. We conclude that germline DND1 mutations are unlikely to contribute significantly to human testicular
\end{abstract}

R. H. Sijmons $(\bowtie) \cdot$ Y. J. Vos · J. C. Herkert .

K. K. Bos $\cdot$ R. M. W. Hofstra

Department of Genetics, University Medical Center Groningen,

University of Groningen, PO Box 30001, 9700 RB Groningen,

The Netherlands

e-mail: r.h.sijmons@medgen.umcg.nl

M. F. L. Holzik · H. J. Hoekstra

Department of Surgical Oncology, University Medical Center

Groningen, University of Groningen, Groningen,

The Netherlands

\section{F. L. Holzik}

Department of Surgery, Medisch Spectrum Twente, Enschede,

The Netherlands

\section{J. E. H. M. Hoekstra-Weebers}

Wenkebach Institute, University Medical Center Groningen,

University of Groningen, Groningen,

The Netherlands germ cell tumor susceptibility. The role of human DND1 in normal physiology and disease, however, is still virtually unknown and it therefore warrants further research.

Keywords Testicular germ cell tumors - DND1 . Germline $\cdot$ Mutation $\cdot$ Familial

\section{Introduction}

A family history of testicular germ cell tumors (TGCT) is a strong risk factor for developing this tumor type [1]. Although low to mild penetrant gene variants have been identified, including cKIT mutations [2], linkage analysis, genome-wide association studies and a candidate gene approach have not yet been successful in identifying highly penetrant germline mutations as the cause of familial TGCT [3-7]. Animal models may help to identify diseasepredisposing mutations in humans, for example, in the 129/ Sv mouse model, a germline mutation in DND1 (Deadend 1) has been shown to cause TGCT [8]. Although the tumors in the mouse model resemble the germ-cell tumors that occur in human infants rather than in adults [9, 10], this does not necessarily indicate that a role for the human orthologue of DND1 in TGCT development would be restricted to that histological subtype. A recent study, the first to analyze germline human DNDI (MIM 609385) in a clinical disorder, therefore screened the gene for mutations in a series of 263 familial and sporadic TGCT patients and found a possibly pathogenic missense p.Glu86Ala mutation in a single case [11]. These findings could neither confirm nor reject a role for germline $D N D 1$ mutations in human TGCT. We therefore searched for germline DND1 mutations in an independent set of sporadic and familial cases of testicular cancer. 


\section{Materials and methods}

Patients

From the 1,113 patients treated for TGCT at the University Medical Center Groningen (UMCG) between 1977 and 2001, we included 30 known familial cases (2.7\% of total) and randomly selected 242 sporadic cases in this study. Familial cases were defined as those patients with one or more reported first- or second-degree relatives with TGCT. In the patients selected for our study, non-seminomas had been diagnosed in 215 of the sporadic cases ( $88 \%$ of all sporadic cases) and in 23 of the familial cases (77\% of all familial cases). Seminomas had been diagnosed in 27 sporadic cases (12\% of all sporadic cases) and in 7 familial cases $(23 \%$ of all familial cases). All patients gave informed consent for this study, which was approved by the UMCG's institutional review board.

\section{Controls}

We tested 210 control chromosomes for any DND1 variant we detected in the patients if it was not reported as a common polymorphism in NCBI dbSNP (http://www.ncbi. nlm.nih.gov/projects/SNP/).

\section{Screening of DND1}

DNA was isolated from peripheral blood lymphocytes using standard methods. Denaturing gradient gel electrophoresis (DGGE, technique reported elsewhere [12] was used to screen DND1 exons 2 and 4, and part of exon 3 for mutations. If there were abnormal bands, the amplicons in question were sequenced. Exon 1 and the 3' site of exon 3 were fully sequenced. DGGE and sequencing primers are listed in Table 1. Reference sequence used: GenBank accession number NM_194249.

\section{Variant analysis}

Any variant detected, excluding the common SNPs, was analyzed in silico using three sequence homology-based programmes: Align-GVGD (http://agvgd.iarc.fr/agvgd_ input.php), PolyPhen (Polymorphism Phenotyping, http:// genetics.bwh.harvard.edu/pph/) and Sorting Intolerant from Tolerance (SIFT, http://blocks.fhcrc.org/sift/SIFT.html). The possible effect on the function of the splice acceptor site, the splice donor site, or creation of a novel splice site was analyzed by using tools from Netgene (http://www.cbs. dtu.dk/services/NetGene2/) and the Berkeley Drosophila Genome Project (http://www.fruitfly.org/seq_tools/splice. html).
Table 1 Primers used for analyzing DND1

Exon 1-F: [M13-F]GTCGCGCTCAGAGCCAATG

Exon1-R: [M13-R]CTTCCTCTGCCCGTTCACC

Product size $362 \mathrm{Bps}$

Exon2-F: [60GC]TCCGCCATTCCAACCTTCCTT

Exon2-R: AAAGGGGGCTGGTGTAGCCG

Product size $258 \mathrm{Bps}$

Exon3A-F: [60GC]CTGACGGTGGCCTTGCATAC

Exon3A-R: GTTGTGCAGCGTGGCGATGG

Product size $311 \mathrm{Bps}$

Exon3B-F: [60GC]ACGAGTTCCGCCTGATGATGACCT

Exon3B-R: AGCCGCGCCTCCTGCAAGCC

Product size $316 \mathrm{Bps}$

DND-1Exon3B + C-F: [M13-F]ACGAGTTCCGCCTGATGATGA

DND-1Exon3B + C-R: [M13-R]AGGTAAGCGGTGGTCGTAG

Product size $527 \mathrm{Bps}$

Exon4A-F: [60GC]GGGTTTAGACCTGCCCTTGT

Exon4A-R: CGGGCCCAGGGCTGCCCAGCTTCATT

Product size $315 \mathrm{Bps}$

Exon4B-F: CGGGCCCGGGCTCGGGCTACCCTGCAG

Exon4B-R: [60GC]TTATGCCCATTCAGGGTGCCTG

Product size 407 Bps

$F$ forward primer

$R$ reverse primer

$[60 \mathrm{GC}]=$ the $\mathrm{CG}$ clamp = CGCCCGCCGCGCCCCGCGCCCGG CCCGCCGCCCCCGCCCGCGCCCGCCGCGCCCCGCGCC

M13-F = CGACGTTGTAAAACGACGGCCAGT

M13-R = CAGGAAACAGCTATGAC

Loss-of-heterozygosity analysis

One patient was found to have an exon 4 missense mutation (see "Results" section) so the paraffin blocks of his tumor were sampled for DNA extraction and subsequent sequencing of the relevant part of DND1 to search for loss of heterozygosity. Three samples were tested: one from his testicular teratoma, one from a neighboring carcinoma in situ, and one from his normal testicular tissue. His healthy parents and two healthy adult brothers were also analyzed for the presence of the missense mutation by direct sequencing.

\section{Results}

In exon 4 of DND1 we observed a single base substitution c.657C > G (p.Asp219Glu) (Fig. 1) in a 31-year-old patient who had been diagnosed with a non-familial case of non-seminoma at the age of 18 years. This patient had an 


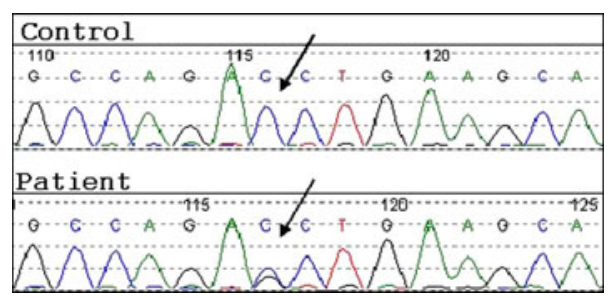

Fig. 1 Germline mutation in patient with testicular teratoma. Sequence of the germline c.657 C > G DND1 mutation detected in the patient (arrow in lower lane)

unilateral small embryonal carcinoma within a field of carcinoma-in-situ. In his paratesticular tissue a small mature teratoma was observed. No loss of the wild type DND1 allele was detected in the patient's tumors or normal tissue samples (results not shown). The germline variant was present in the patient's healthy father and his 2 healthy adult brothers, who had no symptoms or history of cancer. This variant was not detected in the 210 control chromosomes. In silico analysis did not predict a pathogenic effect. Although the amino acid residue at this position is highly conserved through evolution, the substitution falls within the same class of amino acids (both have negatively charged $\mathrm{R}$ groups, Grantham Score 45). The variant is not located in any known functional domain (http://www.ncbi.nlm.nih. gov/Structure/cdd/wrpsb.cgi?seqinput=NP_919225.1). Align GVGD indicated the p.Asp219Glu missense substitution to be non-pathogenic (class $\mathrm{C} 0$, with a Grantham Variation of 61.28 and a Grantham Deviation of 0.00).

Sorting Intolerant from Tolerance (SIFT) analysis indicated this substitution to be neutral, with a tolerance index score of 0.73 (normalized probabilities of $>0.05$ are predicted to be tolerated). Polyphen classified the variant as benign with a PSIC score of 0.498 (scores of $<1.5$ are predicted to be benign). This variant has no effect on the function of the splice acceptor site, nor does it create a novel splice site (Netgene and Berkeley Drosophila Genome Project tools).

In addition to the single case of c. $657 \mathrm{C}>\mathrm{G}$, two other variants were detected in our study population. In exon 3 , the silent mutation c.363T $>\mathrm{C}$ (p.=) was observed in 172 out of 272 patients $(63 \%)$; it was homozygous in 60 out of these 172 patients $(35 \% ; 22 \%$ of all patients). All the 172 patients were also found to carry an intron 3 variant, c. $604+47 \mathrm{G}>\mathrm{A}$. Two other patients only carried this c. $604+47 \mathrm{G}>\mathrm{A}$ variant. Of the $174(172+2)$ patients who carried this c. $604+47 \mathrm{G}>\mathrm{A}$ variant, 59 (34\%; $22 \%$ of all patients) were homozygous for it. The NCBI dbSNP database (http://www.ncbi.nlm.nih.gov/projects/SNP/) reports a high heterozygosity frequency for both variants: 0.499 for c. $363 \mathrm{~T}>\mathrm{C}$ and 0.497 for c. $604+47 \mathrm{G}>\mathrm{A}$, and both are regarded as normal population variants.

\section{Discussion}

We detected a germline missense c.657 C > G (p.Asp219 Glu) DNDl variant in a single TGCT patient with a teratoma. From a clinical point of view, his family history was negative for cancer and the fact that both his father and brothers carry the variant suggests either low penetrance or that the variant makes no contribution to the tumor development. The variant resides outside the RNA binding functional domain of the gene and in silico tests indicated non-pathogenicity. In the TGCT mouse model, homozygous DND1 germline truncating mutations cause total loss of DNDl function. We therefore tested our patient's tumour for loss of the wild type allele. Both alleles were detected in his tumor samples, which rules out the possibility of loss of DNDI function through physical loss of the wild type allele and offers no evidence for pathogenicity of the observed germline missense mutation. However, the existence of a somatic mutation in the wild type allele cannot be excluded. The other variants, c.363T $>\mathrm{C}$ and c. $604+47 \mathrm{G}>\mathrm{A}$, observed frequently in our patients, have been reported as common polymorphisms by NCBI dbSNP and are not therefore suspected of contributing to TGCT development. The p.Glu86Ala $D N D 1$ variant detected in a single TGCT case by Linger et al. [11] was classified by these authors as possibly pathogenic, based on in silico analysis and its absence in controls.

No functional analysis is currently available to assist in classifying DND1 missense mutations. Indeed, very little is known about the role that human DNDI plays in normal physiology or in disease. The gene has been shown to be expressed in a range of normal tissues, including testicular germ cells, Leydig cells, interstitial cells and cells from the seminiferous tubules [13-15]. Generally, little is known about expression of DND1 in cancer, but it might be increased in colorectal adenocarcinoma, leukemia, prostate cancer, squamous cell carcinoma, and papillary thyroid cancer [14]. In addition, DND1 maps to a region (5q31.1) that has been found to be deleted in TGCT [16] and it has been shown to protect the expression of a range of genes from repression by miRNAs in primordial germ cells [17]. A role for germline or somatic DND1 mutations in human cancer, and in particular in TGCT, cannot be ruled out. If it plays a role, its mechanics may well be complicated since the genetics of the DND1-mouse-TGCT-model now appear to be complex [18, 19].

Our study and that of Linger et al. [11] may have a limitation in that they may have underestimated the frequency of $D N D 1$ germline mutations in adult patients. The presence of most large deletions or duplications, deep intronic sequence variants, or variants in the regulatory sequences that might affect gene function could not have been detected with the methods we and Linger et al used to 
analyze the gene. However, the vast majority of germline mutations in the known human tumor syndromes are truncating mutations, or in some cases missense mutations, e.g. in Li-Fraumeni syndrome and Multiple Endocrine Neoplasia type 2 , and these types would have been detected by both studies. It is therefore reasonable to conclude that germline DND1 mutations are unlikely to contribute to tumor development in the majority of testicular germ cell tumors diagnosed in juveniles and adults. Whether this is also true for the very rare group of teratomas and yolk-sac tumors of neonates and infants, that more closely resemble the tumors observed in mice with germline DND1 mutations, remains to be investigated.

Acknowledgments The research was performed with a grant of the $\mathrm{C}$ and $\mathrm{W}$ de Boer Foundation. We thank Professor Harry Hollema, Department of Pathology, University Medical Center Groningen, for tumor samples.

Open Access This article is distributed under the terms of the Creative Commons Attribution Noncommercial License which permits any noncommercial use, distribution, and reproduction in any medium, provided the original author(s) and source are credited.

\section{References}

1. Lutke Holzik MF, Rapley EA, Hoekstra HJ et al (2004) Genetic predisposition to testicular germ-cell tumours. Lancet Oncol 5(6):363-371

2. Rapley EA, Turnbull C, Al Olama AA et al (2009) A genomewide association study of testicular germ cell tumor. Nat Genet 41:807-810

3. Rapley EA, Crockford GP, Teare D et al (2000) Localization to Xq27 of a susceptibility gene for testicular germ-cell tumours. Nat Genet 24(2):197-200

4. Sonneveld DJ, Lutke Holzik MF, Nolte IM et al (2002) Testicular carcinoma and HLA Class II genes. Cancer 95(9):1857-1863
5. Nathanson KL, Kanetsky PA, Hawes R et al (2005) The Y deletion gr/gr and susceptibility to testicular germ cell tumor. Am J Hum Genet 77(6):1034-1043

6. Crockford GP, Linger R, Hockley S et al (2006) Genome-wide linkage screen for testicular germ cell tumour susceptibility loci. Hum Mol Genet 15(3):443-451

7. Lutke Holzik MF, Hoekstra HJ, Sijmons RH (2006) Re-analysis of the $\mathrm{Xq} 27-\mathrm{Xq} 28$ region suggests a weak association of an $\mathrm{X}$-linked gene with sporadic testicular germ cell tumour without cryptorchidism. Eur J Cancer 42(12):1869-1874

8. Youngren KK, Coveney D, Peng X et al (2005) The Ter mutation in the dead end gene causes germ cell loss and testicular germ cell tumours. Nature 435(7040):360-364

9. Looijenga LH, Oosterhuis JW (1999) Pathogenesis of testicular germ cell tumours. Rev Reprod. 4(2):90-100

10. Zhu R, Bhattacharya C, Matin A (2007) The role of dead-end in germ-cell tumor development. Ann N Y Acad Sci 1120:181-186

11. Linger R, Dudakia D, Huddart R et al (2008) Analysis of the DND1 gene in men with sporadic and familial testicular germ cell tumors. Genes Chromosomes Cancer 47(3):247-252

12. Hayes VM, Wu Y, Osinga J et al (1999) Improvements in gel composition and electrophoretic conditions for broad-range mutation analysis by denaturing gradient gel electrophoresis. Nucleic Acids Res 27:e29

13. Su AI, Wiltshire T, Batalov S et al (2004) A gene atlas of the mouse and human protein-encoding transcriptomes. Proc Natl Acad Sci U S A 101(16):6062-6067

14. Gene Expression Omnibus (GEO): http://www.ncbi.nlm.nih.gov/ geo. Cited 13 Oct 2009

15. Symatlas. http://symatlas.gnf.org Cited 13 Oct 2009

16. Mitelman F, Johansson B and Mertens F (Eds.) Mitelman Database of Chromosome Aberrations in Cancer. http://cgap.nci.nih.gov/ Chromosomes/Mitelman Cited 13 Oct 2009

17. Kedde M, Strasser MJ, Boldajipour B et al (2007) RNA-binding protein Dnd1 inhibits microRNA access to target mRNA. Cell 131(7):1273-1286

18. Hammond S, Zhu R, Youngren KK et al (2007) Chromosome X modulates incidence of testicular germ cell tumors in Ter mice. Mamm Genome. 18(12):832-838

19. Lam MY, Heaney JD, Youngren KK (2007) Trans-generational epistasis between Dnd1Ter and other modifier genes controls susceptibility to testicular germ cell tumors. Hum Mol Genet 16(18):2233-2240 